\title{
FLEUVES ET FORÊTS DANS LA GERMANIA DE TACITE: ÉLÉMENTS REPRÉSENTATIFS DE L'ESPACE GERMANIQUE
}

\begin{abstract}
José Mambwinikivuila-Kiaku*
RÉSUMÉ: Deux éléments naturels reviennent régulièrement dans le paysage germanique de la Germania, à savoir: les eaux (cours d'eau, fleuves, marécages, voire lacs) et les immenses forêts. Cette constante dans la Germania signifie tout simplement que Tacite définit le territoire de la Germanie par des images spatiales de la liquidité et de l'insaisissable en raison de l'immensité des forêts. Si les fleuves permettent de charrier leurs eaux jusqu'à l'embouchure avec l'Océan, ils sont cette force qui, dans ces immenses forêts, organise la vie. Comment Tacite représente-t-il ces deux éléments naturels - les fleuves et la forêt -, quels artifices utiliset-il pour mieux les représenter et quelle (s) fonction(s) leur attribue-t-il? Telles sont les questions auxquelles nous allons tenter de répondre dans cet article tout en évitant de longs développements.
\end{abstract}

MOTS-CLÉ: Tacite; Germania; espace; poétique.

\section{RIOS E FLORESTAS NA GERMANIA DE TÁCITO: ELEMENTOS REPRESENTATIVOS DO ESPACO GERMANICO}

RESUMO: Dois elementos naturais surgem regularmente na paisagem germânica da Germania, a saber: as água (cursos d'água, rios, pântanos e mesmo lagos) e as imensas florestas. Essa constante na Germania significa tão-só que Tácito define o território da Germânia por meio de imagens espaciais da liquidez e da inacessibilidade em razão da vastidão das florestas. Se os rios deixam correr suas águas até a desembocadura no Oceano, são eles a força que, nessas imensas florestas, organiza a vida. Como Tácito representa esses dois elementos naturais - os rios e a floresta -; de que artifícios ele se vale para melhor representá-los, e que função(ões) lhes atribui? Estas são as questões que tentaremos responder neste artigo, sem muito estendermo-nos.

PALAVRAS-CHAVE: Tácito; Germania; espaço; poética.
* Département des Lettres et Civilisation Latines. Université Pédagogique Nationale (UPN) de Kinshasa - RD Congo. jmamabwini@yahoo.fr 
$\mathrm{D}$ ans le cadre de nos enseignements sur les «Questions de poétique» organisés au sein de notre département, nous avons été amenés à procéder à une relecture de la Germania de Tacite dans la perspective de cerner la «poétique tacitéenne de l'espace» dans cet ouvrage. ${ }^{1}$ Si l'on tient compte des indices spatiaux et paysagers dans cet ouvrage et en s'intéressant aux circonstances au cœur desquelles ces indices ont été introduits dans le récit tacitéen, on en vient à cette conclusion: Deux éléments naturels reviennent régulièrement dans le paysage germanique de la Germania, à savoir: les eaux (cours d'eau, fleuves, marécages, voire lacs) et les immenses forêts. Les fleuves contribuent à l'organisation sociale et économique des peuples qui habitent sur les rives (ripae), tandis que les forêts constituent des espaces de vie protégés pour les Germains. Ce sont ces deux points que nous allons développer tout au long de cet article.

\section{Les fleuves, ÉlÉments essentiels dans L'ORganisation SOciale ET ÉCONOMIQUE DES GERMAINS}

La Germania débute par une description physique de l'espace germanique dominé par les fleuves, ${ }^{3}$ une description dont chaque phrase apporte une information spécifique à tel ou tel fleuve. Entrant ex abrupto dans le vif du sujet sans présenter ni ses intentions ni proposer un plan, Tacite-géographe commence son étude ethnographique sur les Germains par la présentation générale du territoire germanique: trois phrases suffisent pour nous préciser, non seulement les limites naturelles de la Germanie libre, ${ }^{4}$ mais également ses principales caractéristiques physiques.

Phrase 1. Usant d'une formule qui nous rappelle le début du De Bello Gallico, ${ }^{5}$ comme s'il voulait s'inscrire dans la continuité littéraire de César, Tacite nous apprend que «Germania omnis a Gallis Rhaetisque et Pannoniis Rheno et Danubio fuminibus, a Sarmatis Dacisque mutuo metu

\footnotetext{
${ }^{1}$ Les idées émises dans cette étude seront développées dans un ouvrage en préparation sous le titre de «La poétique de l'espace dans l'œuvre de Tacite: la construction de l'espace singulier dans les opera minora» qui sera publié aux Editions L'Harmattan, à Paris.

${ }^{2}$ L'édition de la Germania utilisée dans cette étude est celle de Tacite, 1983.

${ }^{3}$ Introduisant son article sur «les fleuves dans le récit militaire de Tacite» (cf. Poignault, 2001, p. 414) que l'on peut considérer comme la suite de son autre article paru en 1999-2000 (cf. Poignault, 19992000), Rémy Poignault précise que «Les fleuves (ou cours d'eau en général) sont des éléments non négligeables du cadre chez Tacite; l'auteur fournit parfois sur ceux-ci des renseignements à caractère géographique, qu'il en présente le cours, qu'il les utilise comme repères pour organiser l'espace ou qu'il s'attache à quelques curiosités; et les grands fleuves apparaissent surtout dans une fonction de frontière idéologique et matérielle.» Ces deux articles nous ont été d'une très grande utilité dans le commentaire concernant l'espace germain.

${ }^{4}$ Elle s'étend à partir de la rive droite du Rhin, et non pas des deux provinces instituées avant 90 ap.J.C. par Domitien, Germania inferior et Germania superior, contrées de la Gaule Belgique avoisinant la rive gauche des cours supérieur et inférieur du Rhin.

${ }^{5}$ César, B.G., 1.1: «Gallia est omnis dinisa...»
} 
aut montibus separatur: cetera Oceanus ambit, latos sinus et insularum immensa spatia complectens, nuper cognitis quibusdam gentibus ac regibus, quos bellum aperuit.». ${ }^{6}$ C'est donc en géographe avisé que Tacite essaie de reproduire l'espace physique de la Germanie en prenant comme repères ses frontières naturelles. ${ }^{7}$ Comme si ce territoire était un espace essentiellement aquatique, l'historien souligne l'importance de deux fleuves - le Rhin et le Danube - qu'il considère d'ailleurs comme étant la frontière naturelle séparant trois peuples, et donc trois espaces de vie, à savoir: les Gaulois, les Rhètes ${ }^{8}$ et les Pannoniens. ${ }^{9}$ Il faut avouer que cette première phrase annonce toute la poétique de la construction de l'espace germanique, une poétique basée essentiellement sur la présence et la disposition géographique de deux plus importants fleuves du territoire germanique, le Rhin et le Danube. ${ }^{10}$

Phrases 2 et 3. Comme le ferait un pédagogue, après avoir souligné le rôle que jouent ces deux fleuves dans la délimitation des espaces de vie des peuples germaniques, Tacite poursuit sa description en nous donnant les spécificités de chacun de ces deux fleuves. Les phrases 2 et 3 constituent un véritable tableau hydrographique de la Germanie. La phrase 2 qui fait écho à la tradition ancienne sur l'Océan ${ }^{11}$ contient une évocation au fleuve Rhin: «Rhenus, Rhaeticarum Alpium inaccesso ac praecipiti vertice ortus, modico flexu in occidentem versus,

\footnotetext{
${ }^{6}$ Ger. 1,1. «La Germanie dans son ensemble est séparée des Gaulois, des Rhètes et des Pannoniens par deux fleuves, le Rhin et le Danube, des Sarmates et des Daces par une crainte mutuelle ou des montagnes; le reste est entouré par l'Océan qui presse de vastes péninsules et des îles d'une immense étendue, on y a reconnu, ces temps-ci, des nations et des rois que la guerre a découverts.»

7 À ce propos, O. Devillers (Devillers, 2010, p. 76), note que «la Germania omnis de Tacite est prioritairement considérée du point de vue de ses frontières externes: a Gallis Raetisque et Pannoniis [...], a Sarmatis Dacisque [...] separatur $(G ., 1,1)$. Elle est donc présentée d'emblée pratiquement dans un rapport d'exclusion avec ses voisins. C'est aussi dans ce sens que s'inscrit la conviction de Tacite que les Germains sont de purs autochtones $(G ., 2,1-7)$ et n'ont été mêlés à aucune autre nation, qu'ils constituent en somme une race «qui ne ressemble qu'à elle-même», tantum sui similem gentem $(G ., 4,1-2)$ » ${ }^{8}$ La Rhétie, territoire de cette province romaine, fut soumise par Drusus et Tibère en 16-15 a. C.; elle ouvrait le Tyrol, l'Est de la Suisse et le canton des Grisons. Tacite ne mentionne pas le Norique qui séparait celle-ci de la Pannonie.

${ }^{9}$ La Pannonie s'étendait entre le Danube et la Drave. Elle comprenait l'Ouest de l'actuelle Hongrie et la Croatie. Conquise entre 35 et 9 a.C, elle devint province romaine en 9 a.C. et fut divisée en Pannonie supérieure et inférieure

${ }^{10}$ En rapportant les campagnes militaires transrhénanes de Germanicus dans les Annales (cf. Ann., 1, 59; 2, 14; 2, 22 ), Tacite définit fréquemment le pays des Germains comme l'espace situé entre le Rhin et l'Elbe, les deux fleuves parallèles formant ainsi respectivement les délimitations occidentales et orientales de la Germanie. Cf. Morin, 2008, 23 qui estime que le Rhin, représenté en tant que délimitation du territoire germanique, pourrait également apparaitre comme la limite des régions habitées par les Germains.

${ }^{11}$ Cf. Morin, 2014, p. 104: Selon la représentation ancienne du monde, un vaste océan circulaire entourait les terres habitées, un vaste océan qui, dans sa portion nord, à partir du Rhin, était désigné en latin sous le nom d'Oceanus septentrionalis. Cet Océan septentrional, qui formait la frontière nord de la grande Germanie, correspond évidemment, dans la géographie moderne, à la mer du Nord
} 
septentrionali Oceano miscetur.», ${ }^{12}$ alors que la phrase 3 est consacrée au Danube: «Danunius, molli et clementer edito montis Abnobae jugo effusus, plures populos adit, donec in Ponticum mare sex meatibus erumpat: septimum os paludibus hauritur. ${ }^{13}$

On trouve dans ces deux phrases, la plume d'un 'Tacite géographe' qui décrit, d'une part, le Rhin «avec l'évocation de son point de départ, de son point d'arrivée et du mouvement d'ensemble du fleuve» ${ }^{14}$ et, d'autre part, le Danube comme un fleuve tranquille qui provient d'une source moins abrupte et qui, avant de se jeter dans la mer, se divise en six bras et que le septième se perd dans des marais. Pour Tacite, il n'y a pas de doute: ces deux fleuves sont ce qu'on appelait 'politiquement' à Rome des frontières naturelles. ${ }^{15}$

Lorsqu'on relit minutieusement ces trois phrases qui constituent le tableau paysager initial qui ouvre la Germania, ${ }^{16}$ on s'aperçoit que Tacite oppose les deux fleuves entre eux, «comme s'il obéissait à un impératif rhétorique d'antithèse». ${ }^{17}$ Ce n'est pas cette opposition ${ }^{18}$ qui nous intéresse, mais c'est plutôt le fait de considérer ces deux fleuves comme «point de repère pour organiser l'espace dans son récit», ${ }^{19}$ mieux, «comme points de repère privilégiés

dont l'hydronyme a gardé l'essence même de la nomenclature latine et exprime encore aujourd'hui la position septentrionale de cette mer par rapport au centre européen.

${ }^{12}$ Tacite, Ger. 1, 2 «Le Rhin, jaillissant dans les Alpes Rhétiques d'un sommet inaccessible et abrupt, s'infléchissant quelque peu vers l'Occident, se mêle à l'Océan septentrional.» Dans les Annales 2,6, Tacite nous donne d'autres précisions concernant ce fleuve: Nam Rhenus uno alueo continuus aut modicas insulas circumueniens apud principium agri Bataui uelut in duos amnis dividitur, seruatque nomen et violentiam cursus, qua Germaniam praeuehitur, donec Oceano misceatur : ad Gallicam ripam latior et placidior adfluens (uerso cognomento Vahalem accolae dicunt), mox id quoque uocabulum mutat Mosa flumine eiusque inmenso ore eundem in Oceanum effunditur.»

${ }^{13}$ Tacite, Ger. 1,3. «Le Danube, épanchant ses eaux sur les douces pentes et les calmes hauteurs du Mont Abnoba, rend visite à de nombreux peuples, puis se précipite dans la mer Pontique par six bouches: un septième bras se perd dans les marais.» Commentant cet extrait, R. Poignault (Poignault, 1999-2000, p. 434) nous fait remarquer que «le cours d'eau du Rhin est décrit avec l'évocation de son point de départ, de son point d'arrivée et du mouvement d'ensemble du fleuve, mais on reste dans le domaine de l'épure, sauf pour ce qui est de sa source, qui exprime la force au détriment de tout sens des réalités.

${ }^{14}$ Poignault, 1999-2000, p. 433.

${ }^{15}$ Dans la pensée de bien des Romains, ces limites naturelles avaient été tracées par les dieux dans leur souci d'établir une sorte d'équilibre spatial dans le monde romain. De la sorte, il ne convenait pas de les dépasser. À en croire Laederich (Laederich, 2001, p. 129-130), cette idée de «frontières naturelles» matérialisées par les fleuves pose un problème à la fois idéologique et stratégique.

${ }^{16}$ La Germania s'ouvre par une certitude géographique et se referme par un doute transcendantal où Tacite ne parvient pas à trancher entre tradition, mythe et religion (Ger., 46,6).

${ }^{17}$ Poignault, 1999-2000, p. 431.

${ }^{18}$ Selon Poignault (Poignault, 1999-2000, p. 435), cette opposition se voit aussi d'un point de vue littéraire. Parlant du Danube, Tacite utilise le verbe «erumpere» qui «suggère la violence du flot, pour rendre la manière dont le Danube se jette dans le Pont-Euxin». Quant au Rhin, l'historien préfère l'utilisation du verbe «miscetun».

${ }^{19}$ Poignault, 1999-2000, p. 431. 
dans la narration car ils permettent d'organiser l'espace». ${ }^{20}$ Ce qui nous intéresse ici, c'est le fait que, dans la représentation de l'espace germain, Tacite se sert du Rhin et du Danube comme «principe d'orientation dans la description» ${ }^{21}$ de ce territoire. Bref, ce qui nous intéresse, c'est le fait que, dans la Germanie, Tacite considère ces fleuves, non seulement, comme «des données géographiques et mirabilia», ${ }^{22}$ mais aussi comme des «frontières»" ${ }^{23}$ "perméables», ${ }^{24}$ qui ne sont pas de simples limites symboliques mais qui doivent être considérés aussi dans leur matérialité. ${ }^{25}$

Partant du tableau que Tacite brosse au début de son ouvrage (Ger. 1-3), il apparaît clairement que, pour cet historien, la Germania, en tant qu'entité géographique, est un territoire naturel, non créé par l'homme, car encadré par des délimitations «naturelles» et légitimes que sont les eaux (Océan, fleuves). Si nous devons aller plus loin dans notre analyse, en nous référant aux raisons qui ont poussé Tacite à rédiger rapidement cet opuscule, nous pouvons dire que les trois phrases qui ouvrent la Germania constitueraient un message subliminaire que Tacite lancerait en direction des Gouvernants romains, à savoir: la seule façon de contenir les menaces germaniques et de stabiliser la frontière Nord de l'Empire passe stratégiquement par la domination, c'est-à-dire l'occupation militaire de deux plus importants fleuves du pays, à savoir: le Rhin et le Danube, ${ }^{26}$ fleuves que R. Poignault considère comme «des éléments de définition de l'espace», mieux comme des «points de repère pour organiser l'espace dans son récit». En tout cas, ce n'est pas par un pur hasard de l'écriture que Tacite ouvre sa Germania en mettant en exergue ces deux fleuves qui, de notre point de vue, ont une importance capitale et vitale pour l'ensemble de l'espace germain composé d'une multitude de peuples et donc une multitude d'espaces vitaux.

En effet, en plaçant ces deux fleuves en antéposition de toute la narration, Tacite voulait très probablement nous dire que, le Rhin et le Danube jouent un rôle important dans

\footnotetext{
${ }^{20}$ Poignault, 1999-2000, p. 436.

${ }^{21}$ Pognault, 1999-2000, p. 437.

22 Poignault, 1999-2000, p. 433-442.

${ }^{23}$ Poignault, 1999-2000, p. 442-451.

${ }^{24}$ Poignault, 1999-2000, p. 451-455. Dans la Germania, même si Tacite considère le Rhin comme un «fleuve frontière», il ne le présente jamais comme infranchissable. En effet, le Rhin n’a pas empêché des Gaulois de passer sur la rive opposée (Cf. Ger., 28,1). L’historien évoque aussi les Germains sur la rive gauche du Rhin (Ger., 28,4). Les Ubiens sont la preuve que le Rhin a été un facteur d'échanges et non de division (Ger., 28,5). Les migrations des Cimbres ont également laissé des témoignages de leur passage sur les deux rives (Ger., 37,1). Les Tongres ont été les premiers à traverser le Rhin et à venir s'installer en Gaule (Ger., 2). Le Danube n'est non plus que le Rhin une barrière infranchissable (Ger., 28,3)

${ }^{25}$ Le Rhin, par ex. du côté des Usipens et des Tenctères, constitue par son seul cours un terminus (une borne) suffisant pour séparer l'empire de ces Germains (Ger., 32,1). Cf. Poignault, 2001, p. 446. ${ }^{26}$ L'importance de ces deux fleuves a fait l'objet de plusieurs études dont certaines dédiées aux représentations mythologiques et symboliques du fleuve et d'autres au Rhin et ainsi que la fonction de fleuve-frontière. Sur cette question, outre Morin, 2008, voir aussi Dion, 1993; Vogler, 1992; Vogler, 1997; Trousset, 1993.
} 
l'organisation sociale de l'espace germain de la même manière que le sont, par exemple, le Nil pour l'Egypte, le Tibre pour l'V rbs, l'Euphrate pour l'ancienne Phénicie. D’une manière générale, bien que s'intéressant plus au fleuve Rhin et à son espace, les travaux de S.-Morin, ${ }^{27}$ tout au moins dans les chapitres ou les passages concernant Tacite, ont abondé dans ce sens. Revenons au texte de la Germania pour insister sur le fait que la mise en valeur de ces fleuves ne peut en aucun cas nous étonner. D’ailleurs, comme l'a si bien souligné S.-Morin, ${ }^{28}$ plusieurs auteurs anciens utilisèrent délibérément le fleuve afin de définir, de délimiter et d'organiser l'espace géographique et politique, se servirent de la lisière fluviale de façon à séparer des environnements opposés, octroyèrent au Rhin, surtout le Rhin inférieur, non seulement une fonction protectrice en le présentant comme une barrière défensive et un obstacle, mais également une fonction culturelle, économique, politico-militaire ${ }^{29}$ et sociale.

Au-delà du fait qu'ils constituent des éléments majeurs de la construction du récit tacitéen dans la Germania, des éléments de division de l'espace, mieux, des éléments discriminants privilégiés qui permettent une délimitation et une organisation de l'espace géographique fonctionnelles, bref, éléments de définition de l'espace, ${ }^{30}$ les fleuves de la Germanie, surtout le Rhin, ainsi que leurs rives, sont aussi perçus dans la Germania comme des éléments structurant et organisant la vie sociale des Germains. Lorsqu'on lit ou qu'on analyse cet opuscule sous cet angle, l'on se rend vite compte que toute la poétique des fleuves germaniques tacitéens se développe autour de cette fonction sociale, très symbolique pour un territoire mal connu comme la Germanie. Relisons la Germanie chapitre par chapitre.

\subsection{LES FLEUVES COMME ÉLÉMENTS DE LOCALISATION DES PEUPLES GERMAINS SOCIALEMENT ORGANISÉS}

Dans la Germania, les indications spatiales ou paysagères liées aux fleuves servent à suggérer la localisation géographique des Germani. S’appuyant sur une représentation du Rhin héritée de César, dès le début de son ouvrage (Ger. 1,1), Tacite utilise régulièrement le fleuve comme séparation ethnique et culturelle entre les Germains et les Gaulois. Dans le paragraphe suivant, c'est par rapport au Rhin (Ger. 2,5) que Tacite commence son étude sur l'implantation, mieux, l'occupation des peuples germaniques. Il se sert du fleuve Rhin pour situer et localiser les peuples étudiés. Selon l'auteur de la Germania, c'est sur la rive droite que se sont établies les nations autochtones. Parmi ces nations, Tacite cite les Chattes qui se sont établis dans la partie sud du cours inférieur du fleuve. Dans la Germania 32,1, Tacite nous informe que «proximi Chattis certum iam alueo Rhenum quique terminus esse sufficiat $V_{\text {sipi ac }}$

\footnotetext{
${ }^{27}$ Cf. Morin, 2008 et 2014.

${ }^{28}$ Morin, 2008, p. 13.

${ }^{29}$ Selon elle (Morin, 2008) le Rhin inférieur demeura un secteur majeur d'activités militaires sous les Julio-Claudiens, les conjonctures et les stratégies politico-militaires de l'époque flavienne, à la fin du I ${ }^{\text {er }}$ siècle, entraînèrent un glissement vers le sud des effectifs romains et une diminution de la prééminence des troupes rhénanes au profit de la zone danubienne (cf. Morin, 2008, p. 3).

${ }^{30}$ Pour tous ces aspects des fleuves de la Germanie chez Tacite, cf. Morin, 2008 et Poignault, 1999-2000.
} 
Tencteri colunts. ${ }^{31}$ Pour identifier certains peuples autochtones vivant sur la rive gauche du fleuve au I ${ }^{\text {er }}$ siècle de notre ère, Tacite se sert aussi du fleuve Rhin: il n'hésite pas à employer ouvertement le terme de «Germani cis Rhenum». ${ }^{32}$ L'historien situe les autres nations ou peuples de la Germanie sur la rive occidentale du Rhin supérieur. C'est le cas des Vangions, des Triboques et des Nemètes, ${ }^{33}$ alors que les Tungri sont placés en Germanie inférieure sans toutefois préciser davantage leur positionnement géographique. Selon l'historien latin, cette nation ayant jadis franchi le Rhin aurait été la première à porter le nom de Germains «(...) quoniam qui primi Rhenum transgressi (...) nunc Tungri, tune Germani uocati sint.» (Tacite, Ger., 2). Tous ces peuples étaient socialement organisés au point de créer des espaces de vie viable. Par exemple, plusieurs années après la Germania, dans les Annales, ${ }^{34}$ parlant des Ubiens, en plus de grandes fermes (uillae), des terres labourées (arua) et des villages (uici), Tacite mentionne la présence d'un oppidum Vbiorum - place forte du territoire ubien - et d'une ara Vbiorum - autel des Ubiens. ${ }^{35}$ Cette organisation de vie avait de rapport avec les fleuves, en l'occurrence ici le Rhin. C'est fort probablement en raison de la présence du delta rhénan que les Bataves s'y sont installés. Dans la Germania, Tacite explique que les Bataves étaient "Chattorum quondam populus et seditione domestica in eas sedes transgressus, in quibus pars Romani imperii fierent»: (Ger. 29). ${ }^{36}$ Il faut dire que l'installation des Bataves dans le delta rhénan est une preuve de l'influence du fleuve sur les populations locales et régionales. C'est donc le Rhin qui a favorisé le mouvement migratoire des Bataves. Selon S.-Morin, ce mouvement a non seulement entraîné l'implantation cisrhénane d'une nouvelle entité tribale indépendante, mais encore fut à l'origine de l'intégration batave à l'Empire romain. Ces racines transthénanes sont à nouveau mentionnées par Tacite dans son récit de la révolte des Bataves, alors que l'origine chatte des insurgés et leur antique migration vers le delta sont, plus tard, rappelées par l'historien dans les Histoires. ${ }^{37}$

\footnotetext{
31 «Au voisinage immédiat des Chattes, le cours du Rhin désormais certain et devenu frontière est habité par les Usipes et les tenctères.»

32 Tacite, Ann., I, 56.

${ }^{33}$ Tacite, Ger., 28, 4: «Ipsam Rheni ripam haud dubie Germanorum populi colunt, Vangiones, Triboci, Nemetes.» (Il n'est pas douteux que la rive même du Rhin soit occupée par des peuples germaniques, vangions, triboques; Némèdes.)

${ }^{34}$ Tacite, Ann., 13, 57. Oppidum Vbiorum (Tacite, Ann., 1, 36; 12, 27); ara Vbiorum (Tacite, Ann., 1, 39, 13, 39).

${ }^{35}$ Cf. Morin, 2008, p. 30.

${ }^{36}$ A propos des Bataves, ainsi que nous pouvons le lire dans les Histoires 4,12, les écrits de Tacite sont sans ambiguïté et multiplient les allusions à l'occupation batave de la grande île rhénane (cf. Ger., 29), une île clairement deltaïque, une île «quam mare Oceanus a fronte, Rhenus amnis tergum ac latera circumluit» précise l'historien latin (Hist. 4,12).

${ }^{37}$ Tacite, Hist., 4,12: «Bataui, donec trans Rhenum agebant, pars Chattorum, seditione domestica pulsi extrema Gallicae orae nacua cultoribus simulque insulam iuxta sitam occupauere, quam mare Oceanus a fronte, Rhenus amnis tergum ac latera circumluit.»
} 


\subsection{LE RHIN: ÉLÉMENT IMPORTANT DANS L'ORGANISATION ET L'ENCADREMENT DES ÉCHANGES ÉCONOMIQUES}

Dans plusieurs passages de la Germania, Tacite souligne le fait que le fleuve Rhin a favorisé les échanges commerciaux entre les peuples germains, mais aussi entre ces peuples et les Romains. Ce fleuve a favorisé la création des zones habitées qui se sont organisées socialement au point de créer des zones économiques dynamiques. Il conviendrait de retenir que les franchissements réguliers de ce fleuve ont joué un rôle majeur dans les relations transfrontalières soutenues entre les populations riveraines. Ainsi le souligne d'ailleurs S.Morin, ces franchissements du Rhin ont permis de conserver des contacts diplomatiques, militaires, économiques ou sociaux entre les rives. Garantissant une communication renouvelée entre les deux côtés du Rhin, ces franchissements réguliers assurèrent le maintien d'une cohésion des rives du fleuve et de l'espace frontalier rhénan. ${ }^{38}$

Principal fleuve dans la zone de contacts militaires et diplomatie transrhénane, ${ }^{39}$ placé au cœur d'une zone de convergence économique ${ }^{40}$ et favorisant de multiples contacts sociaux et culturels entre les groupes rhénans, ${ }^{41}$ seul fleuve germain situé au cœur d'un espace frontalier alimenté par des interactions multiples entre les deux rives, le Rhin joue un rôle social très important dans la mesure où, il favorisait, par ses traversées, la circulation humaine accrue par la mise en place des aménagements fluviaux spécifiques ainsi que d'échanges interethniques et des transferts culturels croissants. ${ }^{42}$

\section{LA Forêt TACITÉENNE de LA GERManie: CADRE DE VIE ET ÉlÉMENT DE CARACTÉRISATION IDENTITAIRE DE L'HOMME GERMAIN}

Dans la textualité de la Germania, au-delà de l'étude ethnographique sur les peuples germaniques, la représentation de l'espace s'appuie sur les rapports que les peuples germaniques ont pu établir et entretenir avec les forêts. Fait caractéristique, Tacite les cite sans trop de précisions dans leurs localisations et sans beaucoup de détails descriptifs. Parmi toutes ces forêts, ${ }^{43}$ l'historien cite l'Abnoba (Ger., 1,2) et surtout, la plus importante de toutes, la «saltus Hercynius» (Ger., 30,1).

\subsection{LA «SALTUS HERCYNIUS»: SYMBOLE DE L'ESPACE SYLVESTRE GERMANIQUE}

Forêt mythique de l'antique Germanie dont les légendes en font une description incroyable en raison de son étendue insensée et des êtres la peuplant, s'étendant jusqu'à la

\footnotetext{
${ }^{38}$ Cf. Morin, 2008, p. 51.

${ }^{39}$ Cf. Morin, 2008, p. 77-87.

${ }^{40}$ Cf. Morin, 2008, p. 88-94.

${ }^{41}$ Cf. Morin, 2008, p. 95-106.

${ }^{42}$ Cf. Morin, 2008, p. 95-107.

${ }^{43}$ Dans les Annales, Tacite signale aussi la silua Caesia (Ann., 1,50,1) probablement située dans la région d'Essen, et le saltus Teutoburgensis (Ann., 1,60,3).
} 
Forêt Noire, de la Thuringe et de l'ancienne Bohême, la «saltus Hercynius» allait encore plus loin du côté du levant et se prolongeait dans la Pologne et dans la Hongrie. Nommée Orcynie par Eratosthène, Arcinie par Aristote, cette forêt tire vraisemblablement son nom de César qui, le premier de tous les auteurs latins, a fait mention de la Hercynia silua (cf. B.G., 6,24). D'après les géographes grecs, César avait confondu toutes les forêts et toutes les montagnes de la Germanie. C'est cette vague tradition qui se serait propagée parmi les géographes romains. Ni Pline l'Ancien ${ }^{44}$ ni Tacite ne surent s'en former une idée très précise. On comprend alors pourquoi, dans la Germania, malgré sa réputation, notre historien ne lui accorde pas de description, se contentant de l'expression «saltus Hercynius» (Ger., 30,1).

\subsection{LA FORÊT GERMANIQUE, UN ESPACE À CONNOTATIONS MULTIPLES}

Dans la textualité de la Germania, la forêt joue plusieurs fonctions. Elle intervient comme: a) un cadre de vie loin de la civilisation de l'V rbs, b) un espace transcendantal, et donc sacré, où le mythe se mêle à l'irrationnel dans la mesure où, à certains moments, elle se transforme en «sanctuaire» de quelques divinités vénérées par les Germains, c) un espace protecteur et stratégique contre l'envahisseur romain. Toutes ces fonctions mises ensemble, la forêt tacitéenne devient, in fine, un espace poétique au cœur duquel Tacite évoque, avec tant de dédain, l'immensité territoriale de la Germanie, source de tant de terreurs et de cauchemars pour l'armée romaine. Examinons rapidement toutes ces fonctions avec l'espoir que d'autres chercheurs exploreront cette piste.

2.2.1. Espace physique, la forêt germanique est avant tout représentée en rapport avec l'homme germain. Dans la Germania, sans toutefois lui accorder une importance capitale si ce n'est que pour souligner le caractère barbare des peuples germaniques, Tacite fait de la forêt une représentation sociale, considérant qu'elle est étroitement liée à la vie et à l'existence des Germains. Il faut lire la Germania dans son intégralité pour s'apercevoir de cette représentation. La forêt est l'espace de vie traditionnelle pour le Germain. Elle leur sert d'habitat, ${ }^{45}$ tout au moins dans la partie la moins marécageuse, ${ }^{46}$ de cachette (lieu de retraite ou d'asile) pour eux-mêmes, ${ }^{47}$ mais également pour leurs biens qu'ils enfouissaient sous terre. La forêt leur sert de réservoir de toutes les ressources dont ils ont besoin : elle fournit du bois pour la construction de leurs cabanes, mais également de la nourriture tant le sol germain est globalement stérile.

\footnotetext{
${ }^{44}$ Pline, H.N, 4,14,100, parle de jugum Hercynium, nom d'une chaîne de montagnes.

${ }^{45}$ C'est notamment le cas pour les Chattes. Tacite, Ger., 30,1: «Vltra hos Chatti initium sedis ab Hercynio saltu incolant,non ita effusis ac palustribus locis ut ceterae cinitates in quas Germania patescit.» (Au-delà, les Chattes ont leurs premiers établissements dans la forêt hercynienne, en un pays moins épandu et moins marécageux que ceux des autres cités où se déploie la Germanie.) Voir aussi Ger., 16,1: «Nullas Germanorum populus urbes habitari satis notum est.»

${ }^{46}$ Lorsqu'on analyse les textes relatifs à la campagne de Germanicus dans les Annales, on s'aperçoit que, pour Tacite, la Germanie est aussi un territoire marécageux. Cf. par ex. Ann., 1,61.

${ }^{47}$ Cf. par ex. Tacite, Ger., 46,4: «... buc redeunt imuenes, hoc senum receptaculum.»
} 
2.2.2. Espace transcendantal, la forêt germanique, tout au moins, une partie de celle-ci, apparaît comme un domaine mythique et/ou sacré dans lequel l'homme germain pratiquait des scènes qui, de nos jours, tiendraient de l'irrationnel. Dans la Germania 39,1, par exemple, nous pouvons lire: «Vetustissimos nobilissimosque Sueborum Semnones memorant; fides antiquitatis religione firmatur. Stato tempore in siluam anguriis eiusdemque sanguinis populi legationibus coeunt caesoque publice homine celebrant barbari ritus horrenda primordia.» ${ }^{48}$ Un peu plus loin, plus précisément au chapitre 40,1-2, Tacite nous informe que «Reudigni deinde et Auiones et Anglii et Varini et Eudoses et Suardones et Nuithones fluminibus aut silvis muniuntur. Nec quicquam notabile in singulis, nisi quod in commune Nerthum, id est Terram matrem, colunt eamque intervenire rebus hominum, invehi populis arbitrantur. ${ }^{49}$ Ces deux exemples, parmi tant d'autres, attestent que les Germains se servaient de la forêt comme d'un sanctuaire. La forêt était pour eux un lieu idéal pour s'adonner aux cultes, un lieu sacré pour faire des sacrifices à leurs divinités. Parmi ces divinités, Tacite cite nommément Nerthus, ${ }^{50}$ divinité de la fertilité vénérée d'une façon assez drôle et particulièrement fêtée au printemps. ${ }^{51}$

\subsubsection{Espace stratégique pour les Germains, espace de tant d'horreurs pour} les Romains. C'est probablement cette fonction qui pousse Tacite à manifester une sorte d'aversion à l'endroit de forêts germaniques au point de qualifier l'espace germanique de «terra horrida siluis». ${ }^{52}$ Cette expression traduit la vision tacitéenne, et donc celle de la majorité des Romains, de forêts immenses, denses et démesurément hautes, de forêts infinies se dressant aux marges du monde romain et masquant la totalité des terres. À voir la manière dont il décrit ces forêts dans ses écrits historiques, l'on a de fortes raisons de croire que, comme

\footnotetext{
${ }^{48}$ «Les plus anciens et les plus nobles des Suèves seraient les Semnons; un fait d'ordre religieux confirme ce qu'on croit de leur antiquité. À époques fixées, les peuples de ce nom et de ce sang se rassemblent par députations dans une forêt; les augures des pères, l'effroi des vieux âges l'ont rendue sacrée; et c'est en immolant officiellement un homme qu'ils célèbrent les horribles prémices de rites barbares.» 49 «Ensuite Reudignes, Aviones, Angles, Varins, Eudoses, Suardones et Nuithons ont pour défense des cours d'eau ou des forêts. Aucun de ces peuples ne se distingue des autres par rien de notable, sinon qu'ils ont un culte commun pour Nerthus, c'est-à-dire la Terre-Mère, croient qu'elle intervient dans les affaires des hommes et circule parmi les peuples.»

${ }^{50}$ Les Germains vénéraient aussi Freya, divinité de l'amour et du foyer; Tiwaz qui dirige la foudre et qui patronne les assemblées; Donar don't les bras lancent la foudre. Cf. Riché, 1974. Bien que très ancien, surtout Dumezil, 1939.

${ }^{51}$ Selon Tacite, Ger. 40,2-3: «Est in insula Oceani castum nemus, dicatumque in eo vebiculum, veste contectum; attingere uni sacerdoti concessum. Is adesse penetrali deam intellegit vectamque bubus feminis multa cum veneratione prosequitur. Laeti tunc dies, festa loca, quaecumque adventu hospitioque dignatur.» (Il y a dans une île de l'océan une forêt sainte, et là un char consacré, couvert d'un voile; le prêtre seul a le droit d'y toucher. Il connaît que la déesse est présente dans son sanctuaire et il l'accompagne très respectueusement, traînée par des génisses. Ce sont alors jours de liesse, c'est fête en tous les lieux qu'elle daigne honorer de sa visite et de son séjour.) Notons que, dans toute la littérature latine concernant les Germains, Tacite est le seul à avoir mentionné Nerthus. La brève description qu'il donne de ce culte évoque celui de Cybèle. (Cf. Lucrèce, II, 589-643) et celui de la Mater Magna à Rome.

${ }^{52}$ Nous retrouvons la même expression chez Horace: Germania...horrida (Carm. IV 5, 26).
} 
pour beaucoup de Romains, Tacite les redoutait tant. Quand, dans la Germania 16,4, il écrit que les Germains «solent et subterraneos specus aperire eosque multo insuper fimo onerant, suffugium biemis et receptaculum frugibus, quia rigorem frigorum eius modi loci molliunt, et si quando hostis advenit, aperta populatur, abdita autem et defossa aut ignorantur aut eo ipso fallunt, quod quaerenda sunt», ${ }^{53}$ Tacite entend aussi que ces cavernes deviendront de secrets et dangereux asiles pour les soldats germaniques et concourront à leur système de défense, système méconnu des soldats romains.

Il est probable qu'en rédigeant la Germanie, eu égard à la représentation qu'il fait des forêts germaniques, Tacite avait en mémoire les désastres que les armées romaines y ont déjà subis, désastres qui ont provoqué un sentiment de terreur chez les Romains. ${ }^{54}$ D’un point de vue militaire, les forêts germaniques étaient un milieu oppressant pour les Romains. En tout cas, l'immensité et l'obscurité de ces forêts intimidaient les Romains habitués aux paysages bucoliques de l'Italie. De plus, le traumatisme du désastre de Varus, où les légions romaines avaient été prises en embuscade dans les profondeurs des forêts germaniques, avait probablement conduit Tacite à construire et nous représenter une image négative des étendues sylvestres au-delà du Rhin. Ainsi qu'on peut le constater, pour les décrire, Tacite emploie un vocabulaire conférant un caractère lugubre, sombre et oppressant à ces milieux.

\subsection{LA SPÉCIFICITÉ DE LA FORÊT GERMANIQUE ET LA CARACTÉRISATION IDENTITAIRE DU GERMAIN}

La représentation tacitéenne de l'espace germanique est profondément négative : lorsqu'on rassemble, comme dans un puzzle, tous les détails spatiaux ou paysagers concernant l'environnement naturel germain, et plus précisément la forêt, on aperçoit sans trop de difficulté que la Germania de Tacite est en quelque sorte un tableau éclaté représentant la Germanie comme un milieu hostile, inhospitalier, ingrat et sauvage. Et la phrase de la Germania 5,1, selon laquelle «Terra etsi aliquanto specie differt, in uniuersum tamen aut siluis horrida aut paludibus foedar ${ }^{55}$ apparaît comme un résumé de cette représentation construite autour de deux éléments naturels, à savoir: la «forêt immense» et le «marécage» perçus par les Germains à la fois comme refuge et comme protection contre les Romains. ${ }^{56} \mathrm{Il}$ est essentiel de noter

\footnotetext{
${ }^{53}$ «Ils ont aussi l'habitude de creuser des cavités souterraines et ils les chargent en dessus d'un gros tas de fumier, refuge contre l'hiver et resserre pour les grains: car ils adoucissent la rigueur des froids par ces sortes d'abris, et s'il arrive que l'ennemi survienne, il ravage ce qui est à découvert, mais ce qui est caché et enfoui se fait ignorer ou lui échappe, par cela même qu'il lui faut le chercher.»

${ }^{54}$ Cf. Tacite, Ann., 1, 61-64.

${ }^{55}$ Le pays, en dépit d’une certaine diversité, est cependant, en général, hérissé de forêts ou enlaidi par des marécages.

${ }^{56}$ Ainsi le note S. Morin (Morin, 2014, p. 287-288), suivant les représentations des auteurs grécoromains, les Germains auraient ainsi constamment cherché à entraîner les armées romaines sur des terrains marécageux où non seulement leur haute taille facilitait leurs mouvements, mais encore où leurs armes et leurs techniques de combat, adaptées à ces environnements, leur donnaient un avantage certain: cuirasses et armes légères, longues piques permettant d'atteindre l'ennemi à distance, embuscades et attaques-surprises, facilité à nager et à reconnaître les gués (Tacite, Ann., 1.63-64; Hist.
} 
que, dans la représentation que Tacite fait de l'environnement forestier germanique, l'élément «forêt» est toujours associé à l'élément «marécage». Ce constat s'observe même dans d'autres ouvrages, à l'instar des Annales. ${ }^{57}$

La représentation de l'espace sylvestre germanique, telle qu'elle se dégage de la Germania, est basée sur des topoi dont plusieurs d'entre eux sont issus d'une longue tradition littéraire, mythologique ou scientifique, remontant même, pour certains d'entre eux, à la littérature grecque classique voire aux poèmes homériques, et ont parfois été véhiculés par le biais d'autres arts, au point d'apparaître déjà dans la littérature latine sous forme de clichés, d'images traditionnelles attendues par un lecteur nourri de culture grecque et latine. Et c'est à dessein que Tacite nous présente l'espace forestier germanique comme un environnement hostile, dangereux et inhospitalier. Cette représentation est conforme à la conception romaine de l'altérité germanique, selon laquelle le Germain ressemblait à la Germanie. Ainsi, c'est en voulant donner une image négative du Germain ${ }^{58}$ que Tacite peint le milieu dans lequel il vit.

À y regarder de près, cette peinture éclatée participerait à la définition identitaire de l'homme germain. La poétique tacitéenne du milieu naturel germain, en général, et forestier, en particulier, tendrait à soutenir l'idée selon laquelle le milieu géographique aurait une influence déterminante sur l'identité des peuples et des individus. Si nous relisions la Germania sous cet angle, nous nous apercevrions que les caractéristiques physiques de l'espace forestier germanique renvoient à l'un des traits identitaires du Germain: outre le fait d'être barbare, le Germain qui, selon Tacite, est l'opposé du Romain, ${ }^{59}$ est un peuple sauvage. À l'hostilité et à l'immensité de l'environnement naturel germanique répond son corps gigantesque et puissant. Au début de la Germania, Tacite s'appuie sur le topos ${ }^{60} \mathrm{du}$ Germain au physique très grand et imposant. Comparé à l'immensité de l'espace dans lequel il vit, à l'instar de bien d'autres auteurs latins, Tacite pense que le Germain n'était pas simplement grand, il était très grand. Et cette haute taille caractérisait autant les hommes, que les femmes. ${ }^{61}$ Confronté au climat cruel de son pays, on le considérait endurant au froid; confronté aux terres stériles de son milieu, on le disait indifférent à la faim. ${ }^{62}$ Pour les Romains, le physique germanique répondait à la rudesse de son environnement naturel. ${ }^{63}$

5.14-17). À travers la plume de Tacite, le général Germanicus en arriva finalement au constat que «fundi Germanos acie et iustis locis, iunari siluis,paludibus» (Tacite, Ann., 2,5).

${ }^{57}$ Dans la représentation du site du désastre de Varus, Tacite fait jumeler un décor à la fois de forêts et de marécages. Cf. Ann., 1.61. C'est aussi la même construction que nous observons chez Velleius Paterculus 2.119 et chez Florus, 2.30.

${ }^{58}$ Cf. Devillers, 2010.

${ }^{59}$ Sur cette opposition, cf. Devillers, 2010, p. 75-77.

${ }^{60}$ Concernant le topos du physique germain qui correspond à celui de l'espace dans lequel il vit, cf. Morin, 2014, p. 238-242.

${ }^{61}$ Tacite, Ger., 20.

${ }^{62}$ Tacite, Ger., 4,3.

${ }^{63}$ Cf. Tacite, Ger., 4,3: «laboris atque operum non eadem patientia, minimeque sitim aestumque tolerare, frigora atque inediam caelo soloue adsueuerunt.» (leur endurance n'est pas la même à la peine et au travail; ils ne 
Alors que le caractère transcendantal de la forêt souligne sa religiosité, alors que la fonction stratégique de cette immense forêt présente le Germain comme un guerrier rompu dans l'art militaire, ${ }^{64}$ la fonction stratégique de la forêt étale ses traits de guerrier sauvage.

Devons-nous parler du déterminisme physique chez Tacite? Loin de là. Et pour cause. Cette conception insiste sur le rôle du milieu naturel dans l'organisation et le développement de la société germanique. Ce n'est pas exactement cela qui ressort de la lecture de la Germania. Cette lecture nous renseigne, au contraire, qu'au regard de sa vie privée ou communautaire, l'environnement naturel, comme la forêt, offrirait des possibilités et des virtualités que l'homme germain choisirait d'exploiter ou non. Cela nous amène à dire qu'au lieu d'évoquer le déterminisme physique dans la Germania, mieux vaut parler - qu'on nous permette cette expression - du possibilisme qui offre au Germain la liberté du choix de mener sa vie à sa guise.

Une chose est sûre: la description de la forêt germanique que Tacite nous propose pose le problème du rapport entre l'homme germain et l'espace naturel, rapport qui, en fin de compte, se traduit par une recherche poétique d'harmonie qui peut être sociale, affective ou identitaire. Sur le plan individuel, l'identité germanique entre en résonance avec les espaces naturels dans lesquels il vit et évolue.

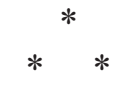

Il nous faut conclure cette étude. Nous avons voulons esquisser quelques idées que nous développerons plus tard et qui fera l'objet d'une publication sous forme d'un livre. Il va sans dire que deux éléments caractérisent l'espace germanique: les eaux et les forêts qui, mis ensemble font de la Germania, en tant qu'espace social et communautaire, est une «terra horrida» (Ger., 5,1) qu'il faudrait absolument maîtrisée si l'on veut réduire la menace germaine. C'est vraisemblablement cette mise en garde ${ }^{65}$ qui expliquerait la rédaction de cet ouvrage quelques mois seulement après la publication de l'Agricola. Pour comprendre cette

\footnotetext{
supportent pas du tout la soif et la chaleur, mais le froid et la faim, ainsi accoutumés par leur ciel ou leur sol.)

${ }^{64}$ Ainsi Devillers, 2010, p. 76: «le Germain ne semble se réaliser pleinement que dans la guerre; autrement, il se montre apathique, préoccupé de dormir et de manger $(G ., 15,1)$. Cette primauté du guerrier, dont le symbole exotique est la framée (elle est mentionnée y compris à propos des mariages; G., 18, 2), indique que, pour Tacite, le problème posé par les Germains est fondamentalement militaire et que c'est sur fond d'antagonisme, toujours latent, avec eux, qu'il livre les résultats de l'enquête qu'il a menée à leur sujet. Il prend néanmoins soin d'articuler cet antagonisme militaire sur d'autres qui l'expliquent et l'éclairent.»

${ }^{65}$ Dans les écrits Tacite, de l'Agricola aux Annales, Tacite a du mal à cacher sa peur et son dédain pour la Germanie. C'est vraisemblablement cette peur et cette attitude dédaignieuse qui dictent sa poétique de l'espace germain, un espace représenté, à quelques exceptions près, sous les traits négatifs. Observez, par ex. la façon dont il décrit le genre de vie que mènent les Germains (Ger., 26) ou encore la manière dont il conseille qu'on envoie du vin à ces peuples pour favoriser leur ivresse (Ger., 23). Relisons, par exemple, les Annales, 1,64-65 dans lesquels il évoque le désastre de Varus.
} 
mise en garde, remottons dans l'histoire. Il est important de rappeler qu'au moment où Tacite écrit la Germania, ${ }^{66}$ Trajan se trouve sur la frontière rhénane. Cette présence mérite quelques explications: il faut remonter dans l'histoire ${ }^{67}$ pour mieux comprendre la présence militaire romaine dans l'espace rhénan. Depuis qu'en l'an 16 avant notre ère, des groupes germaniques transrhénans - des Sugambres, des Usipètes et des Tenctères - avaient franchi le Rhin et attaqué une légion romaine stationnée en Gaule, pour sa sécurité et celle de ses frontières, Rome a exprimé le désir de conquérir les territoires outre-Rhin. Et comme le note Mélissa S.-Morin, ${ }^{68}$ la présence romaine dans la région rhénane devint à cette époque effective et le Rhin inférieur se transforma en zone majeure d'activités militaires dans le cadre des opérations de conquêtes, de domination et de contrôle des peuples germaniques. Pendant près de trente ans, des campagnes militaires furent orchestrées successivement par les généraux romains, à savoir: Drusus, Tibère et Germanicus afin de concrétiser la mainmise romaine sur les territoires transrhénans. L'atteinte de l'Elbe permit d'étendre le monde romain jusqu'à ce fleuve et d'entamer l'organisation d'une nouvelle province. En l'an 7 de notre ère, P. Quintilius Varus obtint ainsi le mandat de structurer la Germanie sur les plans fiscal et judiciaire. Toutefois, après deux années d'administration romaine, alors que Rome croyait le processus d'intégration de la région bien enclenché, trois légions et neuf corps auxiliaires, sous l'égide de Varus, furent anéantis par un peuple autochtone appelé Chérusques. Menés par leur chef Arminius, ces Germains firent plus de vingt mille victimes chez les Romains et infligèrent à Auguste la plus importante défaite de son principat. La Clades V ariana-désastre de Varus - provoqua le repli des troupes romaines dans les districts militaires de la Germanie inférieure et la Germanie supérieure, sur la rive gauche du Rhin, provoquant ainsi une rupture marquée dans la politique germanique de Rome; la stratégie offensive et impérialiste fut remplacée par un positionnement défensif sur le fleuve permettant une stabilisation de l'occupation romaine dans la région rhénane. Malgré l'abandon définitif en 16 de notre ère du projet de conquête de la Germanie et l'échec de l'annexion des territoires à l'est du Rhin, Rome maintint, pendant tout le $\mathrm{I}^{\mathrm{er}}$ siècle, une concentration légionnaire soutenue dans la zone rhénane dans le but de sécuriser les territoires internes et d'assurer une maitrise des déplacements transrhénans des peuples autochtones. Voilà pour l'explication de la présence de Trajan sur la frontière rhénane.

Avant de rentrer à Rome, Trajan procède à une inspection des armées chargées de défendre l'Empire contre les Barbares, sur le Rhin et sur le Danube. En écrivant la Germania, Tacite entendait-il inciter le nouvel empereur à entreprendre un grand projet offensif? Il est difficile de trancher. Est-il que l'histoire des peuples germains, considérée dans sa globalité, nous donne de sérieuses indications pouvant nous aider à expliquer la quintessence de la

\footnotetext{
${ }^{66}$ Dans son Tacite, Grimal, 1990, p. 131-140, a résumé les circonstances qui ont conduit Tacite à rédiger cet ouvrage.

${ }^{67}$ Cf. Velleius Paterculus (II, 97), Suétone (Aug. XXIII, 1) et Dion Cassius (LIV, 20).

${ }^{68}$ Morin, 2008, p. 2-4.
} 
Germania 33,3. ${ }^{69}$ Ainsi que le note P. Grimal, ${ }^{70}$ les pays germaniques sont à l'ordre du jour depuis le règne de Domitien. Les troubles qui s'y produisent périodiquement risquent d'avoir des retentissements à l'intérieur de l'Empire. Mais ces mêmes troubles peuvent être avantageux pour Rome, dans la mesure où ils affaiblissent les nations germaniques et, ainsi, préparent la conquête. Tel semble le sens de l'expression «urgentibus imperii fatis» ${ }^{71}$ par laquelle l'historien déclare que la Fortune ne saurait faire mieux pour Rome que de mettre la discorde parmi ces peuples ennemis «alors que les destins de l'Empire se font pressants».

En tout cas, bien interprétée, la phrase de la Germania 33,3 résume la pensée de Tacite sur ses intentions de publier rapidement la Germania quelques mois seulement après Agricola. Une lecture sérieuse de cet ouvrage montre que, de par tout ce qu'il a écrit, Tacite tendait à œuvrer à la destinée implacable de l'imperium romanum de s'étendre le plus loin possible malgré les obstacles qui pourraient se présenter sur son chemin. Parmi ces obstacles, il y a, bien entendu, la Germanie, ses peuples et son espace, véritable menace pour l'Vrbs et son expansion. D’ailleurs, les menaces sous-entendues dans la Germania 33,3 se sont réalisées: en effet, bien des années après, Rome a dû faire face aux invasions barbares venues du Nord. Et comme c'était la volonté du destin ou plutôt des destins, Rome s'en est sortie victorieusement en peu de temps. En écrivant la Germania, Tacite voulait certainement susciter une prise de conscience chez ses contemporains: face à l'ambition expansionniste, voire impérialiste, toujours grandissante de l'Vrbs, les peuples barbares, malgré leurs dissensions et leurs discordes, représentaient un danger énorme.

En patriote, mais également en historien romanocentriste, Tacite se devait de fournir quelques renseignements sur ces peuples, leurs habitudes et, accessoirement, leur espace. Accessoirement car, à voir le contenu de la Germania, Tacite a accordé une place de choix à l'ethnographie des peuples germaniques, ${ }^{72}$ une ethnographie qui, comme nous l'avons souligné dans une étude récente, a sans nul doute été influencée par le discours sur l'altérité. Les Germains sont un peuple, ou plutôt sont des peuples instables. Leur instabilité est d'autant plus dangereuse pour Rome qu'ils sont passionnés par la guerre (Ger., 14, 3-5). En récapitulant l'histoire des relations entre Rome et les Germains (cf. Ger., 27, 2-6), Tacite voudrait faire prendre conscience aux Romains afin que soit mis en œuvre un projet

\footnotetext{
${ }^{69}$ «Maneat, quaeso, duretque gentibus, si non amor nostri, at certe odium sui, quando urgentibus imperii fatis nibil iam praestare fortuna maius potest quam hostium discordiam».

${ }^{70}$ Grimal, 1994, p. 466.

${ }^{71}$ Malgré les nombreuses recherches et les nombreux commentaires consacrés à ce passage, l'interprétation exacte de «urgentibus imperii fatis» continue de faire l'objet de controverses nourries. Pour la synthèse de discussion, cf. Laederch, 2001, p. 408, note 17. Aussi Devillers, 2010, p. 80. À tout prendre, ainsi le note d'ailleurs Grimal, 1990, p. 136, cette expression exprime un pessimisme prophétique, la pensée d'un esprit inquiet, persuadé que les Germains font peser sur Rome une menace à laquelle il sera impossible d'échapper.

${ }^{72}$ On peut citer les Usipi (Ger., 32), les Tencteri (Ger., 13; Hist., IV, 64), les Chatti (Ger., 32; Ann., I, 56), les Bructeri (Ann., I, 60), les Frisii (Ger., 34; Ann., IV, 73; XIII, 54; Hist., IV, 15), les Ampsiuarii (Ann., XIII, 55), les Vbii (Ger., 37), etc.
} 
stratégique $^{73}$ global susceptible, dans le sens des fata imperii, de réduire à néant la menace que font peser certains Germains ${ }^{74}$ sur la sécurité frontalière au Nord. Finalement, ces Germains, qui sont-ils exactement?

Comme le note M. S. Morin, ${ }^{75}$ sous le vocable «germain» cache une kyrielle de tribus locales aux aspirations concurrentes; les querelles et les alliances entre les peuplades germaniques étaient connues et Rome se plut d'ailleurs régulièrement à attiser les rivalités tribales pour son propre intérêt. Or, malgré cette compréhension romaine des divisions tribales, les représentations sociales des peuples germaniques édifiaient habituellement l'image d'un ensemble ethnique homogène, politiquement cohérent et culturellement uni. À deux endroits $^{76}$ au début de son ouvrage, Tacite nous donne son point de vue sur la Germanie et son peuple, ou plutôt ses peuplades avant de couper court: «Ipse eorum opinionibus accedo, qui Germaniae populos nullis aliis aliarum nationum conubïs infectos propriam et sinceram et tantum sui similem gentem extitisse arbitrantun $:^{77}$ les Germains apparaissaient comme une entité indigène - indigena - n'ayant pas connu de métissages, tous les individus étant liés par une ascendance commune. Malgré leurs nombreuses guerres fratricides, ils étaient souvent considérés comme un bloc politique unitaire, s'exprimant d'une seule voix et s'opposant ensemble à l'envahisseur romain. De tels peuples ${ }^{78}$ étaient donc un danger pour l'V rbs et son empire.

\footnotetext{
${ }^{73}$ Sur cette question précisément chez Tacite, cf. Laederich, 2001.

${ }^{74}$ Pour cette menace, il convient de préciser que ce ne sont pas tous les Germains. Comme le souligne P. Grimal (Grimal, 1994, p. 466); certains peuples germains acceptent la civilisation et, en même temps, l'amitié des Romains. Il en va ainsi pour les Bataves, les Ubiens de Cologne, exemples d'assimilation réussie. Ces Germains-là peuvent être utiles aux Romains par l'exemple qu'ils donnent de vertus maintenant oubliées.

${ }^{75}$ Morin, 2014, p. 233.

${ }^{76}$ Cf. Tacite, Ger., 2, 1 et 2, 5.

77 Tacite, Ger., 4,1 «Pour moi, je me range à l'opinion de ceux qui pensent que les peuples de la Germanie, pour n'avoir jamais été souillés par d'autres unions avec d'autres tribus, constituent une nation particulière, pure de tout mélange et qui ne ressemble qu'à elle-même.»

${ }^{78}$ L'image du Germain correspond aux caractéristiques et aux représentations de l'espace germanique véhiculées par la littérature ancienne, mieux par la littérature greco-latine: un espace sauvage dans lequel vivent des hommes sauvages et barbares. Dans cette littérature, le Germain est avant tout cette personne qui vient de Germanie, celui qui vient de cet immense territoire au-delà du Rhin que les Romains, incapables de l'appréhender dans son entièreté, ont nommé la Germania. C'est pour cette raison, nous semble-t-il, qu'avant de parler de chaque peuple ou tribu qui y habite, Tacite a choisi de nous présenter le tableau physique de ce territoire. Comme qui dirait, c'est la Germanie, terre sauvage qui a engendré les Germains, peuples sauvages et barbares. Lorsque, dès les premières lignes de son célèbre ouvrage, César, l'une des sources de Tacite, écrit que les Germains, voisins des Gaulois, sont ceux «qui trans Rhenum incolunt» (B.G. 1,1), n'est-ce pas qu'il caractérise les Germains non pas en fonction d'une culture ou d'une langue, mais bien en fonction d'un espace géographique? Ce rapport de l'identité du Germain liée à son espace de vie, c'est-à-dire à la Germanie donne à cet espace une autre signification au-delà de sa signification géographique. Sur l'image du Germain dans la Germania, cf. Devillers, 2010. Subsidiairement aussi Ridé, J. (1977), L’Image du Germain dans la pensée
} 
La seule façon de réduire ce danger consiste à l'occupation stratégique de leur espace en amenant les Romains à devenir les «maîtres» de leurs eaux, donc de leurs fleuves, et de leurs forêts. Stratégie difficile à mettre en place.

\section{BIBLIOGRAPHIE}

ALONSO-NÚÑEZ, J. M. Significación de la Germania de Tácito. Zephyrus, v. 25, p. 473-478, 1974.

BREEZE, D. J.; DOBSON, B. Roman Milirary Deployment in Nord England. Britannia, v. 16, p. 1-19, 1985.

DEVILLERS, O. Image du Germain dans la Germanie de Tacite. Vita latina, v. 182, p. 75-84, 2010.

DION, R. Rhenus bicornis. R.E.L., v. 42, p. 469-499, 1993.

DUMEZIL, G. Mythes et dieux des Germains. Paris, 1939.

DUPONT, F. “En Germanie c'est-à-dire nulle part”. Rhétorique de l'altérité et rhétorique de l'identité: l'aporie descriptive d'un territoire barbare dans la Germanie de Tacite. In: ROUSELLE, A. (ed.). Frontières terrestres, frontières célestes dans l'Antiquité. Paris: Presses universitaires de Perpignan, 1995, p. 189-219.

GIUA, M.A. Paesaggio, natura, ambiente comme elementi strutturali nella storiografia di Tacito. $A N R W$ II, v. 33, n. 4, p. 2879-2902, 1991.

GRIMAL, P. Tacite. Paris: Fayard, 1990.

GRIMAL P. La littérature latine. Paris: Fayard, 1994.

KRÖNER, H.-O. Tacitus, Germania 2, 1 Germaniam... informem... asperam...tristem. LEC, v. 50, p. 99-113, 1982.

LAEDERICH, P. Les limites de l'Empire: les stratégies de l'impérialisme romain dans l'œuvre de Tacite. Paris: Economia, 2001.

LUND, A.A. Kritischer Forschungsbericht zur 'Germania' des Tacitus. $A N R W$, II, v. 33, n. 3, p. 2109-2124, 1991.

MORIN, M. S. Le Rhin et l'espace frontalier rhénan au Ier siècle de notre ère: entre représentation d'une frontière romaine et situation frontalière d'un environnement fluvial. Université Laval, 2008. (Mémoire de maîtrise)

MORIN, M. S. Le delta du Rhin de César à Julien. Représentations d'un environnement deltaïque aux frontières du monde romain. Universités Laval et Franche-Comté, 2014. (Thèse de doctorat)

et la littérature allemandes: de la redéconverte de Tacite à la fin du XVf siècle: contribution à l'étude de la genèse d'un mythe. Thèse - Université de Paris IV, 1976. Paris, Diffusion H. Champion, 2 v. 
MUCH, R. Die Germania des Tacitus, Heildelberg, 1967.

POIGNAULT, R. Les Fleuves de la Gaule et des provinces avoisinantes chez Tacite comme éléments de définition de l'espace. Caesardunum, v. XXXIII-XXXIV, p. 431-455, 1999-2000.

POIGNAULT, R. Les fleuves dans le récit militaire tacitéen. Latonmus, v. 60, p. 414432, 2001.

RICHÉ, P. Les invasions barbares. Paris: PUF, 1974.

RIDÉ, J. L'Image du Germain dans la pensée et la littérature allemandes: de la redécouverte de Tacite à la fin du XVI siècle: contribution à l'étude de la genèse d'un mythe. Paris: Diffusion H. Champion, 1997.

RIVES, J. B. Germania. In: PAGAN, V. E. (ed.). A Companion to Tacitus. Oxford: WileyBlackwell, 2012. p. 45-61.

TACITE. La Germanie. Texte établi et traduit par Jacques Perret. $4^{\text {me }}$ tirage revu et corrigé. Paris: Les Belles Lettres, 1983.

THOMAS, R. F. The Germania as Literary Text. In: WOODÙA, A. J. (ed.). The Cambridge Companion to Tacitus. Cambridge: Cambridge University Press, 2012, p. 59-72.

TROUSSET, P. La notion de "ripa” et les frontières de l'Empire. In: PICQUET, F. (ed.). Le Fleuve et ses métamorphoses. Actes du colloque international tenu à l'Université Lyon 3-Jean Moulin, les 13, 14 et 15 mai 1992. Paris: Didier Érudition, 1993, p. 141-152.

VOGLER, C. Le Rhin de César à Théodose. In: RACINE, P. (ed). Flewves, rivières et canaux dans l'Europe occidentale et médiane. Actes du Colloque de Strasbourg 1 et 2 décembre 1995). Nancy: CNDP/CRDP, 1997, p. 85-120.

VOGLER, C. L'Image de deux fleuves frontières de l'Empire romain au IVe siècle: le Rhin et le Danube dans Ammien Marcellin. In PICQUET, J. (ed.). Le Fleuve et ses métamorphoses. Actes du colloque international tenu à l'Université Lyon 3-Jean Moulin, les 13, 14 et 15 mai 1992. Paris: Didier Érudition, 1993, p. 153-159.

Recebido em: 13 de outubro de 2016. Aprovado em: 1 de novembro de 2016. 